\title{
METRIC SPACES WITH SUBEXPONENTIAL ASYMPTOTIC DIMENSION GROWTH
}

\author{
NARUTAKA OZAWA
}

\begin{abstract}
We prove that a metric space with subexponential asymptotic dimension growth has Yu's property $A$.
\end{abstract}

Let $X$ be a metric space. We denote by $B(x, S)$ the closed ball with center at $x$ and radius $S$. Recall that $X$ has Yu's property $A$ if there is a net of functions $\zeta^{n}: X \rightarrow \ell_{1}(X)$ such that

(1) $\zeta_{x}^{n} \geq 0$ and $\left\|\zeta_{x}^{n}\right\|=1$ for all $n$ and $x$,

(2) for every $n$, there is $S_{n}>0$ such that $\operatorname{supp} \zeta_{x}^{n} \subset B\left(x, S_{n}\right)$ for all $x \in X$,

(3) for every $R>0$, one has $\lim _{n} \sup \left\{\left\|\zeta_{x}^{n}-\zeta_{y}^{n}\right\|: d(x, y) \leq R\right\}=0$.

(See Remark 2 below on this definition.) Let us recall from $[\mathrm{Dr}$ the notion of asymptotic dimension growth. Consider a cover $\mathcal{U}$ of $X$. It is uniformly bounded if the diameters of the elements of $\mathcal{U}$ are uniformly bounded. The Lebesgue number $L(\mathcal{U})$ is the infimum of those $\lambda \geq 0$ satisfying the condition that every subset $B \subset X$ of diameter at most $\lambda$ is contained in some element of $\mathcal{U}$. The multiplicity $m(\mathcal{U})$ is the maximal number of elements of $\mathcal{U}$ with a nonempty intersection. Finally, the asymptotic dimension function of $X$ is an extended integer valued function $\operatorname{ad}_{X}$ defined for $\lambda \in[0,+\infty)$ by

$$
\operatorname{ad}_{X}(\lambda)=\min \{m(\mathcal{U}): \mathcal{U} \text { a uniformly bounded cover of } X \text { such that } L(\mathcal{U}) \geq \lambda\}-1 .
$$

The metric space $X$ is said to have subexponential asymptotic dimension $\operatorname{growth}$ if $\operatorname{ad}_{X}$ has subexponential growth. See [Dr] for details. In this short paper, we prove the following.

Theorem 1. A metric space $X$ with subexponential asymptotic dimension growth has Yu's property A.

This extends Dranishnikov's theorem ([Dr]) that polynomial asymptotic dimension growth implies Yu's property $A$. In [DS, Dranishnikov and Sapir introduce the notion of dimension growth and prove that Thompson's group $F$ has exponential dimension growth. At the end of [DS], they ask whether expanders have exponential dimension growth and whether subexponential dimension growth implies coarse embeddability. As

Date: August 08, 2011.

2000 Mathematics Subject Classification. Primary 54F45; Secondary 20F69, 51 F99.

Key words and phrases. coarse metric space, asymptotic dimension, Yu's property A. 
subexponential dimension growth implies subexponential asymptotic dimension growth (the converse is also likely, but it is not clear at the moment of writing and in particular it is not known whether Thompson's group $F$ has exponential asymptotic dimension growth), the above theorem answers both problems in the affirmative. (See [Wi] for the relationships among Yu's property $A$, coarse embeddability, and expanders.)

Proof. The averaging method used in this proof is borrowed from [Ka]. We will reproduce it here for the reader's convenience. Let us fix $n$ and construct $\zeta^{n}: X \rightarrow \ell_{1}(X)$. Take a uniformly bounded cover $\mathcal{U}=\left\{U_{i}: i \in I\right\}$ of $X$ such that $L(\mathcal{U}) \geq 6 n$ and $m(\mathcal{U})=$ $\operatorname{ad}_{X}(6 n)+1$. For every $i \in I$, we choose $x_{i} \in U_{i}$ and let $J: \ell_{1}(I) \rightarrow \ell_{1}(X)$ be the corresponding contraction sending $\delta_{i}$ to $\delta_{x_{i}}$. For every $x \in X$ and $k \in \mathbb{N}$, we set $S_{x}(k)=$ $\left\{i \in I: B(x, k) \subset U_{i}\right\}$. Then, for every $x, y \in X$ and $R \in \mathbb{N}$ with $d(x, y) \leq R$, one has

$$
S_{x}(k+R) \subset S_{x}(k) \cap S_{y}(k) \subset S_{x}(k) \cup S_{y}(k) \subset S_{x}(k-R) .
$$

For every non-empty finite subset $S$, we denote by $\xi_{S}=|S|^{-1} \chi_{S}$ the uniform probability measure on $S$ and note that

$$
\left\|\xi_{S}-\xi_{T}\right\|=2\left(1-\frac{|S \cap T|}{\max \{|S|,|T|\}}\right) .
$$

Now, we define

$$
\eta_{x}^{n}=\frac{1}{n} \sum_{k=n+1}^{2 n} \xi_{S_{x}(k)} \in \ell_{1}(I) \text { and } \zeta_{x}^{n}=J\left(\eta_{x}^{n}\right) \in \ell_{1}(X)
$$

It follows that $\operatorname{supp} \zeta_{x}^{n} \subset B\left(x, \sup _{i} \operatorname{diam}\left(U_{i}\right)\right)$ for all $x$. It is left to verify the condition 3 , Let $R \in \mathbb{N}$ be given and consider $n>R$. Then, for every $x, y \in X$ with $d(x, y) \leq R$, one has

$$
\left\|\eta_{x}^{n}-\eta_{y}^{n}\right\| \leq \frac{1}{n} \sum_{k=n+1}^{2 n}\left\|\xi_{S_{x}(k)}-\xi_{S_{y}(k)}\right\| \leq \frac{2}{n} \sum_{k=n+1}^{2 n}\left(1-\frac{\left|S_{x}(k+R)\right|}{\left|S_{x}(k-R)\right|}\right)
$$

But since

$\frac{1}{n} \sum_{k=n+1}^{2 n} \frac{\left|S_{x}(k+R)\right|}{\left|S_{x}(k-R)\right|} \geq\left(\prod_{k=n+1}^{2 n} \frac{\left|S_{x}(k+R)\right|}{\left|S_{x}(k-R)\right|}\right)^{1 / n}=\left(\frac{\prod_{k=2 n-R+1}^{2 n+R}\left|S_{x}(k)\right|}{\prod_{k=n-R+1}^{n+R}\left|S_{x}(k)\right|}\right)^{1 / n} \geq m(\mathcal{U})^{-2 R / n}$

(here we used the fact that $1 \leq\left|S_{x}(k)\right| \leq m(\mathcal{U})$ for all $k \leq 2 n+R$ ), this implies

$$
\left\|\zeta_{x}^{n}-\zeta_{y}^{n}\right\| \leq\left\|\eta_{x}^{n}-\eta_{y}^{n}\right\| \leq 2\left(1-m(\mathcal{U})^{-2 R / n}\right) .
$$

Since $m(\mathcal{U})=\operatorname{ad}_{X}(6 n)+1$ has subexponential growth in $n$, we obtain the conclusion. 
Remark 2. The definition of Yu's property $A$ used in this paper is different from the original one (particularly when $X$ does not have bounded geometry). However, since each $\zeta^{n}$ constructed in the proof is such that $m(\mathcal{U}) ! n \zeta_{x}^{n}$ are integer-valued for all $x$, Yu's property $A$ in its original form $([\mathrm{Yu}])$ also follows. See [Wi] for more information.

Acknowledgment. This research was done while the author was visiting at Texas A\&M University in the summer 2011 for "Workshop in Analysis and Probability" supported by NSF. He gratefully acknowledges the kind hospitality and stimulating environment provided by TAMU and the program organizers. The author would like to thank Professor M. Sapir for beautiful lectures on coarse embeddings during the workshop, in which he asked if subexponential asymptotic dimension growth implies Yu's property $A$. The author was partially supported by JSPS and Sumitomo Foundation.

\section{REFERENCES}

[Dr] A. Dranishnikov; Groups with a polynomial dimension growth. Geom. Dedicata 119 (2006), 1-15.

[DS] A. Dranishnikov and M. Sapir; On the dimension growth of groups. J. Algebra, to appear. arXiv:1008.3868.

[Ka] V. Kaimanovich; Boundary amenability of hyperbolic spaces. Discrete geometric analysis, 83-111, Contemp. Math., 347, Amer. Math. Soc., Providence, RI, 2004.

[Wi] R. Willett; Some notes on Property A. Limits of graphs in group theory and computer science, 191-281, EPFL Press, Lausanne, 2009.

[Yu] G. Yu; The coarse Baum-Connes conjecture for spaces which admit a uniform embedding into Hilbert space. Invent. Math. 139 (2000), 201-240.

RIMS, KYOTO UnIVERsity, 606-8502

E-mail address: narutaka@kurims.kyoto-u.ac.jp 